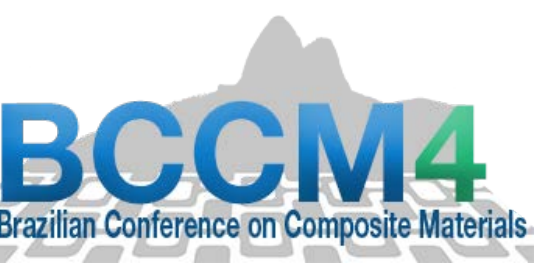

\title{
HYBRID COMPOSITES: EXPERIMENTAL AND ANALYTICAL ASSESSMENT AIDED BY ONLINE SOFTWARE
}

\author{
$\underline{\text { Eduardo A. W. de Menezes }}{ }^{(1)}$, Mário J. Kunz Filho ${ }^{(2)}$, Frederico Eggers ${ }^{(3)}$ and Sandro C. \\ Amico(3) $^{(3)}$ \\ (1) PROMEC, Federal University of Rio Grande do Sul, Brazil \\ (2) Department of Computational Sciences, Federal University of Rio Grande do Sul, Brazil \\ (3) PPGEM, Federal University of Rio Grande do Sul, Brazil
}

https://doi.org/10.21452/bccm4.2018.01.04

\begin{abstract}
Hybrid composites are commonly applied to obtain tailor-made properties due to the additive or synergistic effect between matrix and fibers, offering a wide range of properties that could not be achieved with a single fiber. However, addition of more than one fiber makes the mechanical behavior of hybrid composites more complex, and classical approaches such as rule of mixtures must be reconsidered when evaluating their mechanical properties using micromechanical theories. The aim of this work is to present an online software tool for researchers and designers, where different models for evaluating mechanical and thermal properties of hybrid composites were incorporated, allowing the user to quickly assess them based on the fibers and matrix properties. The developed software is web-based and was programmed and designed with markup and programming languages such as Python, HTML, JavaScript and MySQL, and also enable analysis of single fiber composites (short and long fibers, aligned or random), particulate composites and nanocomposites. In order to verify the available models, an experimental study was carried out by producing an epoxy composite with glass and carbon fibers at different relative fiber volume fraction. The samples were submitted to longitudinal and transverse tensile and in-plane shear testing to produce experimental values for Elastic moduli, Poisson's ratio and shear modulus of the intraply hybrid composite. Good correlation was found between experimental and analytical approaches considering the adopted assumptions, especially for the Chamis and Chou models.
\end{abstract}

\section{INTRODUCTION}

Hybrid composites are commonly referred as a matrix reinforced by at least two distinct fibers, aiming to provide a synergistic effect between the multiple fibers [1]. According to Ashori and Nourbakhsh [2], for the hybrid effect to appear, fibers of different mechanical properties are required, forming also interfaces with different properties. Hybridization may offer a more cost-effective way to meet design requirements compared to traditional engineering 
materials, since it allows composites to reach tailor-made properties [3], balanced thermal stability, strength and stiffness, better toughness, and fatigue and impact resistance [4]. In order to reach balanced properties, it is essential to accurately equalize the volume fraction of matrix and fibers and evaluate the composite mechanical properties or estimate them through micromechanics. However, hybrid effects may not be accurately modelled by the rule-ofmixtures (ROM) [5], increasing the complexity of closed-form solutions. Despite that, micromechanical equations for hybrid composites (HC) provide accurate solutions for predicting mechanical properties, yielding results quite close to the ones obtained through finite element modelling [6].

Unlike isotropic materials, experimental characterization of composite materials is expensive and time consuming due to many variables involved [7]. Aiming to provide an easy way to quickly compute hybrid composite properties from micromechanics, equations found in literature for these material classes were compiled and fed to the MECH-Gcomp software developed by the group. The program interface was built using html customized with CSS. Language JavaScript was used, allowing the scripts to be executed by the user without passing by the server, increasing the development speed. The calculations and server side of the web application used Python, a widely used language, with large online community, high abstraction level and easy programming for the interaction with other languages and libraries. Interaction between Python and framework Django was crucial for a robust web development, together with the program Mysql for MECH-Gcomp database management.

The software MECH-Gcomp already has many different micromechanical approaches, validated with experimental data for nanocomposites [8], particulate composites [9], short [10] and long [11] single fiber composites. The present work aims to compare micromechanical equations available in the literature with experimental results for hybrid glass + carbon/epoxy composites, at different relative fiber volume fractions, to verify their robustness in predicting the composite properties.

\section{MATERIALS AND METHODS}

\subsection{Mathematical Models}

Due to different simplifications and assumptions of different micromechanical models (no voids, neglection of interface, perfect alignment of fibers, etc.), MECH-Gcomp software presents all available options and lets the user decide which one will be chosen. It incorporates in its code several models to compute engineering constants, mechanical strengths and thermal properties of HC. Regarding longitudinal Young modulus $\left(E_{1}\right)$ and Poisson ratio $\left(v_{12}\right)$, Rule of Mixture (ROM) is able to provide accurate results, even for hybrid composites [6, 12].

$$
\begin{aligned}
& E_{1}=E_{1, f 1} V_{f 1}+E_{1, f 2} V_{f 2}+E_{m} V_{m} \\
& v_{12}=v_{13}=v_{12, f 1} V_{f 1}+v_{12, f 2} V_{f 2}+v_{m} V_{m}
\end{aligned}
$$

For the other engineering constants, Banerjee (2014) [6] extended the semi-empirical models proposed by Halpin (1976) [13], applying the following set of equations:

$$
E_{2}=E_{3}=E_{m}\left[\frac{1+\xi\left(\eta_{f 1} V_{f 1}+\eta_{f 2} V_{f 2}\right)}{1-\eta_{f 1} V_{f 1}-\eta_{f 2} V_{f 2}}\right] ; \eta_{f 1}=\frac{\frac{E_{1, f 1}}{E_{m}}-1}{\frac{E_{1, f 1}}{E_{m}}+\xi} ; \eta_{f 2}=\frac{\frac{E_{1, f 2}}{E_{m}}-1}{\frac{E_{1, f 2}}{E_{m}}+\xi}
$$




$$
G_{12}=G_{13}=G_{m}\left[\frac{1+\xi\left(\eta_{f 1} V_{f 1}+\eta_{f 2} V_{f 2}\right)}{1-\eta_{f 1} V_{f 1}-\eta_{f 2} V_{f 2}}\right] ; \eta_{f 1}=\frac{\frac{G_{12, f 1}}{G_{m}}-1}{\frac{G_{12, f 1}}{G_{m}}+\xi} ; \eta_{f 2}=\frac{\frac{G_{12, f 2}}{G_{m}}-1}{\frac{G_{12, f 2}}{G_{m}}+\xi}
$$

where $E_{2}$ is the transverse Young Modulus, $G_{12}$ is the in-plane shear modulus, $V$ is the volume fraction, and $\xi$ is a parameter associated with fiber geometry (for circular fibers its value is 1.165 in Equation (3) and 1.01 in Equation (4)). Subscripts $m, f 1$ and $f 2$ designate matrix, primary and secondary fiber, respectively.

Regarding longitudinal tensile strength, it is assumed that the composite will fail when its first constituent reaches its ultimate strain, as depicted in Equation (5), where $\sigma_{1}{ }^{\mathrm{T}}$ is the longitudinal tensile strength and $\varepsilon$ is the lower ultimate strain among the composite constituents.

$\sigma_{1}^{T}=E_{1} \varepsilon$

According to Chamis (1980) [12], HC properties can be achieved by splitting it on primary composite (PC) and secondary composite (SC), where the first is reinforced by fiber 1 and the second by fiber 2, with the matrix proportionally distributed. The methodology applied to compute these composite properties is analogous to that applied for single-fiber composites, as depicted in Chamis (1989) [14]. Then, the HC engineering constants and strengths are evaluated according to a new ROM. Equation (6) shows how to obtain the transverse Young modulus and, by substituting $E_{2}$ for $G_{12}$ in Equation (6), one can obtain the HC shear modulus.

$$
\begin{aligned}
& E_{2, P C}=\frac{E_{m}}{1-\sqrt{V_{f, P C}}\left(1-\frac{E_{m}}{E_{2, f 1}}\right)} ; \quad E_{2, S C}=\frac{E_{m}}{1-\sqrt{V_{f, S C}}\left(1-\frac{E_{m}}{E_{2, f 2}}\right)} \\
& E_{2}=E_{2, P C} V_{P C}+E_{2, S C} V_{S C}
\end{aligned}
$$

The model presented by Chou [15] splits the composite producing a high modulus (HM) and a low modulus (LM) composite. The equations proposed by Chamis [12] are used to compute their engineering constants, but the final properties are obtained by using the following equations:

$$
\begin{aligned}
& E_{1}=E_{1, H M} V_{H M}+E_{1, L M} V_{L M} ; \quad v_{12}=v_{12, H M} V_{H M}+v_{12, L M} V_{L M} ; \\
& E_{2}=\frac{E_{2, H M}}{1+V_{L M}\left(\frac{E_{2, H M}}{E_{2, L M}}-1\right)} ; \quad G_{12}=\frac{G_{12, H M}}{1+V_{L M}\left(\frac{G_{12, H M}}{G_{12, L M}}-1\right)}
\end{aligned}
$$

\subsection{Experimental Tests}

Hybrid and non-hybrid composite laminates were produced by using carbon/epoxy and glass/epoxy towpregs from TCR Composites, Toray T700-12K-50C and 158B-AB-450, respectively, both pre-impregnated with UF3369 epoxy resin system. The flat laminates were manufactured by dry filament winding using a KUKA 140 L100 robot integrated with peripheral control systems from MF TECH. The equipment delivery eye is able to process up to four towpregs simultaneously allowing the manufacturing of hybrid composites with different compositions. The composite design was performed in the CadWind software and the data containing all the winding parameters were entered. These parameters are converted into a simulated model for manufacture optimization. The simulation is then processed and converted into a robot programming language. 
Hoop flat laminates were produced from the deposition of the fiber tows on top of a stainlesssteel mandrel $\left(327 \times 228 \times 12 \mathrm{~mm}^{3}\right)$, as shown in Figure 1 . Five laminates with different relative contents of reinforcing material were produced, with up to 4 simultaneous tows. Two of the five laminates are composed of a single fiber, glass or carbon. Table 1 shows the amount of tows used and the final thickness of the composite. The laminate ID designates the carbon and glass fiber volume fractions. After winding, the material was cured in a hot hydraulic press under six ton at $120{ }^{\circ} \mathrm{C}$ for $4 \mathrm{~h}$. After that, the system was cooled to room temperature, and the mandrel unscrewed to extract the flat composite.

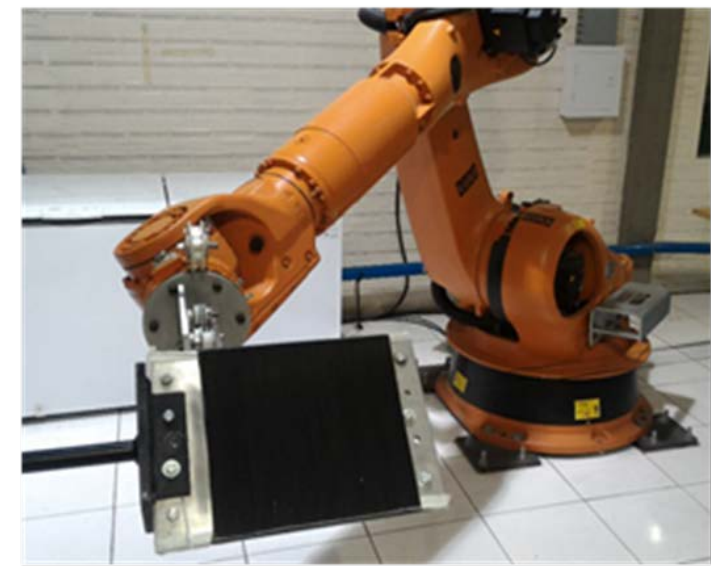

(a)

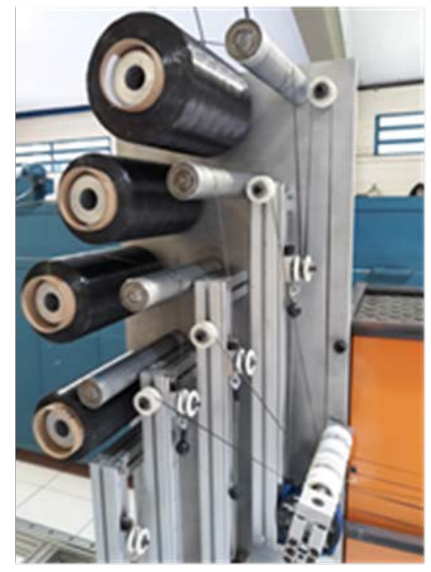

(b)

Figure 1: (a) Manufacturing of a flat laminate by filament winding and (b) arrangement with four simultaneous tows.

Table 1: Specification of the manufactured laminates.

\begin{tabular}{ccccc}
\hline \multirow{2}{*}{ Laminate ID } & \multicolumn{2}{c}{ Tows } & Layc & \multirow{2}{*}{ Mean thickness } \\
\hline 0C:64G & 0 & Glass/epox & & 2.01 \\
19C:44G & 1 & 3 & 3 & 2.25 \\
36C:30G & 1 & 1 & 3 & 2.00 \\
49C:13G & 3 & 1 & 3 & 2.27 \\
61C:0G & 2 & 0 & 3 & 2.06 \\
\hline
\end{tabular}

*C-carbon, G-glass

The unidirectional composites were cut using a CNC router machine, longitudinally and transversely to the fiber direction. The specimens were then sanded and the dimensions measured.

All the experiments were performed in an Instron Universal machine model 3382 with a 100 $\mathrm{kN}$ load cell. Tensile tests were performed according to ASTM D3039-17 at constant speed (2 $\mathrm{mm} / \mathrm{min}$ ) and using two analogical extensometers. Seven specimens for each composite were tested to obtain elastic moduli and Poisson's ratio. For transverse tensile tests, length of the samples was $27 \mathrm{~mm}$ shorter than suggested by the standard due to the limited dimensions of the plates. The shear properties were characterized using ASTM D7078-12, which is recommended for high-modulus fiber-reinforced composite materials. Four samples were tested at a speed rate of $2 \mathrm{~mm} / \mathrm{min}$. Strain gages of the rosette type KFG-5-120-D17-11 from KYOWA were used to enable calculation of the in-plane shear modulus, $G_{12}$. 
Fibers and resin mechanical properties are compiled in Table 2, along with densities $(\rho)$ and weight fraction in towpregs $\left(W_{f}\right)$. Some of the data shown in this table were extracted from the literature [16] when not provided by the manufacturer.

Table 2: Properties of fibers and resin of the towpregs.

\begin{tabular}{r|c|c|c}
\hline Prope & Glass fiber 158B-AB-4 & Carbon fiber T & Epoxy resin UF3369-1 \\
\hline $\boldsymbol{E}_{\mathbf{1}} \mathbf{( G )}$ & 72.4 & 230 & 3.10 \\
$\boldsymbol{E}_{\mathbf{2}} \mathbf{( G )}$ & 72.4 & 15.0 & 3.10 \\
$\boldsymbol{v}_{\mathbf{1 2}}$ & $0.200^{*}$ & $0.200^{*}$ & $0.350^{*}$ \\
$\boldsymbol{G}_{\mathbf{1 2}} \mathbf{( G}$ & $3.08^{*}$ & $14.7^{*}$ & $1.24^{*}$ \\
$\boldsymbol{\rho} \mathbf{( k g} / \mathbf{r}$ & 2580 & 1800 & 1180 \\
$\boldsymbol{W}_{\boldsymbol{f}} \mathbf{( 9}$ & 75.1 & 70.3 & - \\
\hline
\end{tabular}

*Extracted from [16]

\section{RESULTS AND DISCUSSION}

Equations (1)-(7) were used to evaluate the five different composites depicted in Table 1. Figures 2 and 3 present the analytical results from the micromechanics models compared to the experimental data, and the relative deviations are depicted in Table 3.

ROM could accurately predict the $E_{1}$ value, including the HC. While the models from Banerjee [6] and Chamis [12] could predict with relative good accuracy $E_{2}$ and $G_{12}$, the Chou model [15] deviated significantly from the measured values.

Regarding $v_{12}$, none of the models could adequately fit the measured values. This may have occurred due to incorrect input values adopted for fibers and resin, which refer to generic data. The models proposed by Banerjee [6] and Chamis [12] yield generally intermediate values between the composite with fiber 1 and the composite with fiber 2, while Chou [15] model acts in a conservative way, applying ROM with "-1" exponent to compute the final HC properties, yielding final properties similar to the LM composite. 

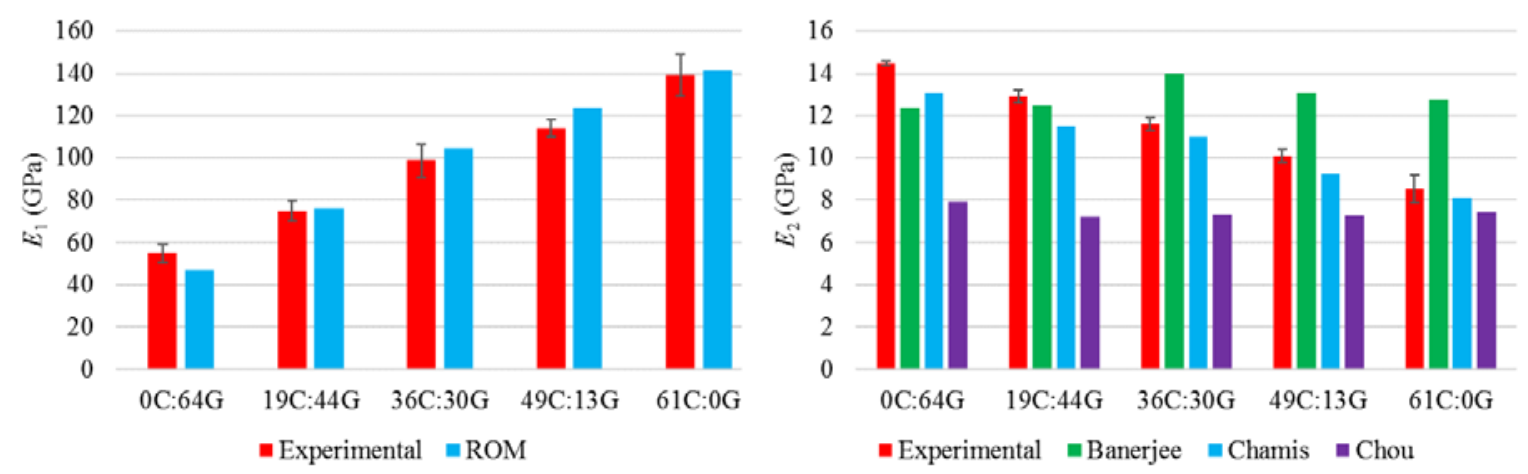

Figure 2: $E_{1}$ and $E_{2}$ for the five different composite configurations.
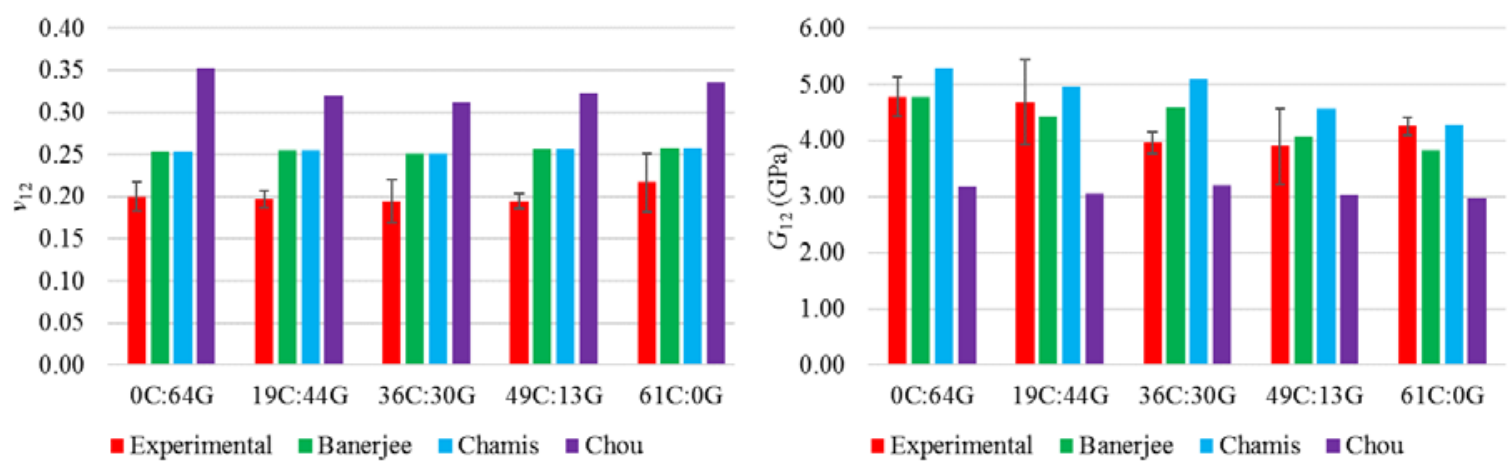

Figure 3: $v_{12}$ and $G_{12}$ for the five different composite configurations.

Table 3: Error between experimental data and micromechanics models.

\begin{tabular}{|c|c|c|c|c|c|c|}
\hline Deviatid & Composi & 100 & 25C:7 & 50C:5 & $75 \mathrm{C}: 2$ & 1001 \\
\hline$E_{1}(\%$ & ROM & 14.3 & 2.00 & 5.7 & 8.2 & 1.5 \\
\hline \multirow{3}{*}{$E_{2}(\%$} & Banerjø & 14.9 & 3.2 & 20.3 & 29.2 & 50.0 \\
\hline & Chami & 9.80 & 11.0 & 5.3 & 8.6 & 4.5\{ \\
\hline & Chou & 45.4 & 44.1 & 36.7 & 27.9 & 12.9 \\
\hline \multirow{3}{*}{$G_{12}(\%$} & Banerje & 0.2. & 5.1 & 15.6 & 4.61 & 10.3 \\
\hline & Chami & 10.4 & 5.99 & 28.4 & 17.1 & 0.71 \\
\hline & Chou & 33.4 & 34.8 & 19.1 & 22.0 & 30.0 \\
\hline \multirow{3}{*}{$v_{12}$} & Banerje & 27.0 & 29.4 & 28.4 & 31.7 & 18.8 \\
\hline & Chami & 27.0 & 29.4 & 28.4 & 31.7 & 18.8 \\
\hline & Chou & 76.0 & 61.9 & 60.0 & 65.6 & 54.8 \\
\hline
\end{tabular}

*Deviations below $15 \%$ have been highlighted for easier comparison.

\section{CONCLUSIONS}

Micromechanical models for hybrid composite materials obtained in the relevant literature were implemented on an online software, allowing its users to quickly compute composite mechanical properties based on fiber and matrix types and volume fractions. In order to verify the model's accuracy, experimental tests were performed for five different configurations.

Considering the already mentioned assumptions and simplifications, and the difficulties in obtaining some materials properties, results were in good agreement with experimental data. Relative to $E_{1}, E_{2}$ and $G_{12}$, micromechanics models showed a deviation of about $10 \%$, except for the Chou model. Poisson's ratio could not be accurately predicted by any model. Thus, it is 
$4^{\text {th }}$ Brazilian Conference on Composite Materials. Rio de Janeiro, July $22^{\text {nd }}-25^{\text {th }}, 2018$

possible to conclude that two of the micromechanical models can be applied for predicting hybrid composites properties in a satisfactory level as first estimates.

\section{ACKNOWLEDGEMENTS}

The authors gratefully acknowledge the National Council for Scientific and Technological Development (CNPq) and UFRGS for the undergraduate scholarships.

\section{REFERENCES}

[1] Kalantari, M., Dong, C. and Davies, I.J., 'Multi-objective analysis for optimal and robust design of unidirectional glass/carbon fibre reinforced hybrid epoxy composites under flexural loading', Comp. P. B: Eng. 84 (2016) 130-139.

[2] Ashori, A., Nourbakhsh, A., 'Hybrid composites from waste materials', J. of Pol. And Env. 18 (1) (2010) 65-70.

[3] Thakur, V. K., Thakur, M. K. and Pappu, A. 'Hybrid Polymer Composite Materials - Properties and Characterization’, 1st Edn (Woodhead Publishing, Duxford, 2017).

[4] Ferrante, L., Tirillò, J., Sarasini, F., et. al., 'Behaviour of woven hybrid basalt-carbon/epoxy composites subjected to laser shock wave testing: Preliminary results', Comp. Part B: Eng. 78 (2015) 162-173.

[5] Henry, J., Pimenta, S., 'Modelling hybrid effects on the stiffness of aligned discontinuous composites with hybrid fibre-types', Comp. Sci. and Tech. 152 (2017) 275-289.

[6] Banerjee, S., Sankar, B. V., 'Mechanical properties of hybrid composites using finite element method based micromechanics', Comp. Part B: Eng. 58 (2014) 318-327.

[7] Kaw, A. K., 'Mchanics of Composite Materials', 2nd Edn (CRC Press, Boca Raton, 2006).

[8] Rodrigues, G., Menezes, E. A. W., Angrizani, C. C., Amico, S. C., 'Evaluation of Nanocomposite properties assisted by software', in '3rd Brazilian Conference on Composite Materials', GramadoRS, Brazil, 2016.

[9] Angrizani, C. C., Menezes, E. A. W., Muller, A. A., Luz, F. F., Amico, S. C., 'Uso de software acadêmico MECH-Gcomp para ensino de compósitos particulados’, Ver. De Ens. De Eng. 36 (1) (2017) 13-23.

[10] Menezes, E. A. W., Angrizani, C. C., Smidt, R., Amico, S. C., 'Propriedades mecânicas de compósitos com fibras curtas aleatórias com auxílio de software online', in ' $13^{\circ}$ Congresso Brasileiro de Polímeros’, Natal-RN, Brazil, 2015.

[11]Luz, F. F., Angrizani, C. C., Muller, A. A., Sarti, A. C. G., Amico, S. C., 'Study of the Properties of unidirectional composites using a dedicated online software', in '2nd Brazilian Conference on Composite Materials’, São José dos Campos-SP, Brazil, 2014.

[12] Chamis, C. C., Sinclair, J. H., 'Mechanics of Intraply Hybrid Composites - Properties, Analysis and Design', Pol. Comp. 1 (1) (1980) 7-13.

[13] Halpin, J. C., Kardos, J. L., 'The Halpin-Tsai equations: A review article’, Pol. Eng. And Sci. 16 (1976) 344-352.

[14]Chamis, C. C., 'Mechanics of Composite Materials: Past, Present and Future', J. of Comp. Tech. and Res., 11 (1) (1989) 3-14.

[15]Chou, T., 'Microstructural design of fiber composites', 1st Edn (Cambridge University Press, New York, 1992).

[16]Hinton, M. J., Kaddour, O. S., Sodden, P. D., 'Failure criteria in fibre reinforced polymer composites: the world-wide failure exercise'. 1st edn. (Elsevier, Oxford, 2004). 